\title{
Induction and maintenance of DNA methylation in plant promoter sequences by apple latent spherical virus-induced transcriptional gene silencing
}

\section{Tatsuya Kon and Nobuyuki Yoshikawa*}

Plant Pathology Laboratory, Faculty of Agriculture, Iwate University, Morioka, Japan

\section{Edited by:}

Nobuhiro Suzuki, Okayama University, Japan

\section{Reviewed by:}

Jeremy Thompson, Cornell University, USA

Masamichi Nishiguchi, Ehime

University, Japan

${ }^{*}$ Correspondence:

Nobuyuki Yoshikawa, Plant Pathology Laboratory, Faculty of Agriculture, Iwate University, 3-18-8 Ueda, Morioka 020-8550, Iwate, Japan e-mail: yoshikawa@iwate-u.ac.jp
Apple latent spherical virus (ALSV) is an efficient virus-induced gene silencing vector in functional genomics analyses of a broad range of plant species. Here, an Agrobacteriummediated inoculation (agroinoculation) system was developed for the ALSV vector, and virus-induced transcriptional gene silencing (VITGS) is described in plants infected with the ALSV vector. The cDNAs of ALSV RNA1 and RNA2 were inserted between the cauliflower mosaic virus $35 \mathrm{~S}$ promoter and the NOS-T sequences in a binary vector pCAMBIA1300 to produce pCALSR1 and pCALSR2-XSB or pCALSR2-XSB/MN. When these vector constructs were agroinoculated into Nicotiana benthamiana plants with a construct expressing a viral silencing suppressor, the infection efficiency of the vectors was 100\%. A recombinant ALSV vector carrying part of the $35 \mathrm{~S}$ promoter sequence induced transcriptional gene silencing of the green fluorescent protein gene in a line of $N$. benthamiana plants, resulting in the disappearance of green fluorescence of infected plants. Bisulfite sequencing showed that cytosine residues at $\mathrm{CG}$ and $\mathrm{CHG}$ sites of the $35 \mathrm{~S}$ promoter sequence were highly methylated in the silenced generation zero plants infected with the ALSV carrying the promoter sequence as well as in progeny. The ALSV-mediated VITGS state was inherited by progeny for multiple generations. In addition, induction of VITGS of an endogenous gene (chalcone synthase-A) was demonstrated in petunia plants infected with an ALSV vector carrying the native promoter sequence. These results suggest that ALSV-based vectors can be applied to study DNA methylation in plant genomes, and provide a useful tool for plant breeding via epigenetic modification.

Keywords: apple latent spherical virus, virus vector, agroinoculation, TGS, DNA methylation, epigenetic modification

\section{INTRODUCTION}

In plants, small RNAs play an important role in the RNA silencing pathway, which interferes with gene expression and also acts against viral infection (Baulcombe, 2004; Kon and Ikegami, 2009). Small RNAs also have an epigenetic mechanism that results in transcriptional gene silencing (TGS) of an endogenous gene through DNA methylation (Law et al., 2010). DNA cytosine methylation is an important epigenetic marker for TGS and controls development and gene expression, and also acts in genome defense against molecular parasites (e.g., viruses, transposons; Liu et al., 2009; Zemach et al., 2010). In plants, the cytosine at CG, $\mathrm{CHG}$, and $\mathrm{CHH}$ sites (where $\mathrm{H}$ is $\mathrm{A}, \mathrm{C}$, or $\mathrm{T}$ ) can be methylated by DNA methyltransferases (Cokus et al., 2008; Law and Jacobsen, 2010). RNA-directed DNA methylation (RdDM), an epigenetic process in plants, requires a trigger for the production of RNAs, including transcripts of endogenous RNA-dependent RNA polymerases and viral/viroid replication intermediates. In an initial step of RdDM, aberrant RNAs are converted into double-stranded RNA (dsRNA), and then this dsRNA is processed by a Dicer-like protein to generate small RNAs (Pontes et al., 2006; Wierzbicki et al., 2008). Small RNAs are loaded onto Argonaute (AGO) proteins and an AGO-RNA complex directs de novo DNA methylation at the target DNA loci (Wierzbicki et al., 2009; Gao et al., 2010). In Arabidopsis, two maintenance classes of DNA methyltransferases [METHYLTRANSFERASE 1 (MET1) and CHROMOMETHYLASE 3 (CMT3)] catalyze cytosine methylation at a CG site and at $\mathrm{CHG} / \mathrm{CHH}$ sites, respectively, whereas DOMAINS REARRANGED METHYLTRANSFERASE 2 (DRM2) is a member of the de novo class of DNA methyltransferases, and is required both for maintenance and initiation of DNA methylation (Cao et al., 2003; Chan et al., 2005). Thus, RdDM induces epigenetic modifications of homologous sequences, and in the gene promoter region, suppresses gene expression at the transcriptional level. Virus-induced transcriptional gene silencing (VITGS) is one of the newer methods for induction of epigenetic modifications in plants (Kanazawa et al., 2011). In plants, DNA methylation can be induced by infection with viruses or viroids. VITGS suppresses gene expression at the level of transcription in the nucleus; moreover, targeting non-coding regions (e.g., gene promoters) results in methylation that is inherited, whereas targeting coding regions also results in methylation, but is not inherited (Jones et al., 2001).

Apple latent spherical virus (ALSV) is a member of the genus Cheravirus in the family Secoviridae. It has a bipartite singlestranded genome (RNA1 and RNA2) encapsidated into three 
capsid proteins (Vp25, Vp20, and Vp24; Li et al., 2000; Le Gall etal., 2007). ALSV infects a broad range of plant species and does not induce any obvious symptoms in most host plants (Igarashi etal., 2009). ALSV vector can effectively induce systemic virus-induced gene silencing (VIGS) because ALSV spreads extensively into growing regions of infected plants (Igarashi et al., 2009; Yamagishi et al., 2011). VIGS down-regulates gene expression in a sequence-specific manner following infection with viral vectors carrying fragments of plant genes (SenthilKumar and Mysore, 2011). VIGS can rapidly allow identification of a loss-of-function phenotype. Therefore, a modified ALSV vector is used for analysis of VIGS of endogenous genes in plants (Yaegashi etal., 2007; Igarashi etal., 2009; Yamagishi and Yoshikawa, 2009). Construction of the ALSV RNA2 vector, containing the cauliflower mosaic virus (CaMV) 35S RNA promoter and nopaline synthase terminator (NOS-T) sequence cloned into a pUC-based plasmid, was previously described ( $\mathrm{Li}$ etal., 2004). Although these viral cDNAs were infectious, the infectivity is not high enough for direct inoculation to many plant species. Cloned ALSV cDNAs first need to be inoculated into Chenopodium quinoa plants by mechanical inoculation, and then the virus propagated in C. quinoa plants is used as an inoculum for target plants. Agroinoculation methods have been established to introduce many plant viruses into plant tissues because Rhizobium radiobacter (previously Agrobacterium tumefaciens) can easily introduce viral genomes and foreign genes into plants by means of a simple inoculation method (Vaghchhipawala et al., 2011).

In this study, two ALSV RNA2 vectors were first cloned into a binary plasmid vector, i.e., one that allows a target sequence to be inserted between multiple cloning sites in the coding region of ALSV RNA2, as previously reported (Li et al., 2004), and other modified vectors that allow a target sequence to be inserted in the 3'-non-coding region of ALSV RNA2. Then we developed an agroinoculation system of ALSV-based vectors that are highly infectious in experimental host Nicotiana benthamiana plants. We also report that an ALSV vector carrying part of the $35 \mathrm{~S}$ promoter sequence was able to induce TGS of the green fluorescent protein (GFP) gene in a transgenic line of $N$. benthamiana plants, and the ALSV-mediated VITGS state was inherited by progeny for multiple generations. Induction of VITGS of an endogenous gene (chalcone synthase- $A$ ) was also demonstrated in petunia plants infected with an ALSV vector carrying the native promoter sequence.

\section{MATERIALS AND METHODS CONSTRUCTION OF ALSV-BASED VECTORS FOR VIGS/VITGS}

Apple latent spherical virus vectors (pEALSR1 and pEALSR2), previously constructed using the CaMV 35 S promoter and the NOS-T in the modified pE18PGT plasmid (Li et al., 2004), were used in this study. The ALSV RNA cDNAs containing the CaMV 35S promoter and the NOS-T sequences were cloned in the binary vector pCAMBIA1300 (Hajdukiewicz et al., 1994) for transformation as described below.

First, the hygromycin phosphotransferase (HPT) gene was removed from pCAMBIA1300 by digestion with Xho I, and then blunt-ended with T4 DNA polymerase. For constructing the ALSV RNA1 vector, an $\sim 0.5 \mathrm{~kb} B a m$ HI-Eco RI fragment containing ALSV RNA1 3'-cDNA including poly-A sequences and the NOS-T from pEALSR1 was cloned into pCAMBIA1300, from which was removed the HPT gene to generate pCAR1-3-NT. A second fragment released from pEALSR1, an $\sim 1.9 \mathrm{~kb}$ Hind IIIXba I fragment containing the CaMV 35S promoter and the ALSV RNA1 5'-cDNA, was cloned into pCAR1-3-NT to generate pCCaMAR1-5/3-NT. Finally, the third fragment released from pEALSR1, an $\sim 6.2 \mathrm{~kb}$ Sac I-Bam HI fragment containing ALSV RNA1 cDNA, was cloned into modified pCCaMAR1-5/3-NT to generate pCALSR1 (Figure 1A).

For constructing the ALSV RNA2 vector, an $\sim 0.9 \mathrm{~kb} K p n \mathrm{I}-E c o$ RI fragment containing ALSV RNA2 $3^{\prime}$-cDNA including poly-A sequences and the NOS-T from pEALSR2 was cloned into pCAMBIA1300 as described above to generate pCAR2-3-NT. A second $\sim 2.2 \mathrm{~kb}$ Hind III-Bam HI fragment containing the CaMV $35 \mathrm{~S}$ promoter and the ALSV RNA2 $5^{\prime}$-cDNA was cloned into pCAR23 -NT to generate pCCaMAR2-5/3-NT. Finally, the third $\sim 1.8 \mathrm{~kb}$ Bam HI-Spe I fragment containing ALSV RNA 2 cDNA was cloned into modified pCCaMAR2-5/3-NT to generate pCALSR2-XSB (Figure 1A).

For constructing the modified ALSV RNA2 vector, four restriction enzyme sites, Mlu I-Nco I, Mlu I-Sal I, Nco I-Sal I, or Sal I-Mlu I, were introduced into pCALSR2-XSB immediately downstream of the translation stop codon of the $108 \mathrm{~K}$ open reading frame using artificial gene synthesis technology to generate pCALSR2-XSB/MN, pCALSR2-XSB/MS, pCALSR2-XSB/NS, and pCALSR2-XSB/SM (Figure 1A).

To construct the ALSV RNA2 vector carrying the phytoene desaturase (PDS) gene of N. benthamiana, the sequence of the PDS gene was amplified by RT-PCR using the specific $\mathrm{P} 1 / \mathrm{P} 2$ primer pair (Table 1). The PCR product was cloned and sequenced. The cloned DNA was digested with Xho I and Bam HI, and then the digested PDS cDNA fragment was cloned into pCALSR2-XSB, which had been digested with Xho I and Bam HI, to produce pCALSR2XSB:NbPDS. To construct the ALSV RNA2 vector carrying the petunia $C H S-A$ gene, the coding sequence of the $C H S$ - $A$ gene (Morita et al., 2012) was amplified by RT-PCR using the specific P3/P4 primer pair (Table 1). The PCR product was cloned and sequenced. The cloned DNA was digested with Sal I and Bam HI, and then the digested $C H S$ - $A$ cDNA fragment was cloned into pCALSR2-XSB, which had been digested with Xho I and Bam HI, to produce pCALSR2-XSB:CHS-A.

\section{CLONING OF RNA SILENCING SUPPRESSORS}

The P19 gene of tomato bushy stunt virus (accession number M21958) was synthesized by artificial gene synthesis (FASMAC Co. Ltd., Atsugi, Japan), and then digested with Xba I and Sac I and cloned into the pBIN3 binary vector (kindly provided by Professor Masato Ikegami) to produce pBIN3:P19. Binary plasmids expressing silencing suppressors $2 \mathrm{~b}$ of cucumber mosaic virus (CMV)-pepo (pBE2113:2b; Yaegashi et al., 2007), AC2 of tomato mosaic Havana virus (pBIN3:AC2, kindly provided by Professor Robert Gilbertson), $50 \mathrm{KP}$ of apple chlorotic leaf spot virus (pBE2113:50K; Yaegashi et al., 2007), and HC-Pro of potato virus Y (pBIN61:HC-Pro, kindly provided by Professor David Baulcombe) were used. 
A PCALSR1

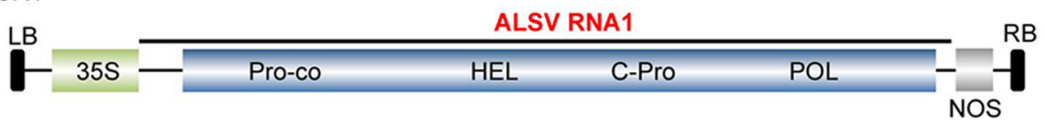

PCALSR2-XSB

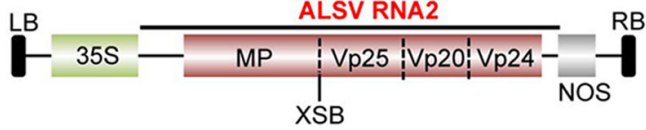

pCALSR2-XSB/MN, MS,NS, or SM

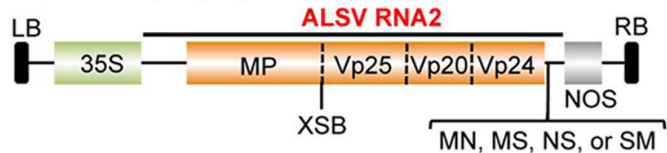

B

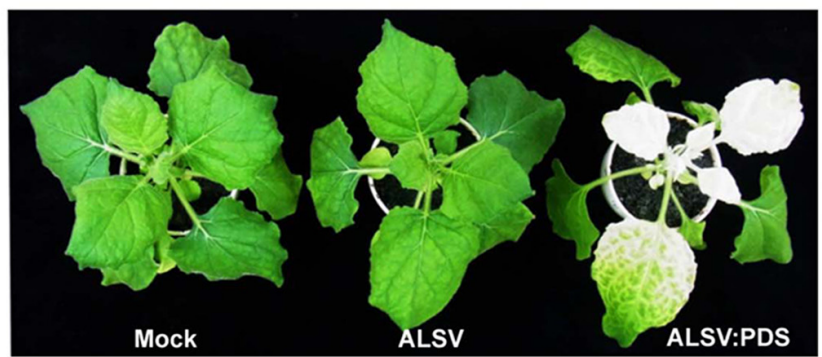

FIGURE 1 | Schematic representation of infectious clones of ALSV RNAs and infectivity of ALSV-based vector in $\boldsymbol{N}$. benthamiana plants. (A) The infectious ALSV RNA1 and RNA2 cDNA sequences (Li etal., 2004) were respectively, introduced between the CaMV 35S RNA promoter (35S) and the nopaline synthase terminator (NOS) within the left and right borders (LB and RB) of the PCAMBIA1300 binary vector to produce pCALSR1 and pCALSR2-XSB. Restriction sites (Mlu I-NCO I [MN], Mlu I-Sal I [MS], Nco-Sal I [NS], and Sal I-Mlu I [SM]) were respectively, introduced into pALSR2-XSB immediately downstream of the translation stop codon of the $108 \mathrm{~K}$ open reading frame to generate pCALSR2-XSB/MN, pCALSR2-XSB/MS, pCALSR2-XSB/NS, and pCALSR2-XSB/SM. The target genes can be cloned into the Xho I, Sma I, and Bam $\mathrm{HI}$ (XSB) restriction sites between the MP and Vp25 duplicate cleavage sites or into the MN, MS, NS, or SM restriction sites immediately downstream of the translation stop codon of the the $108 \mathrm{~K}$ open reading frame. The open reading frames of ALSV represent the protease co-factor (Pro-co), NTP-binding helicase (HEL), cysteine protease (C-Pro), RNA polymerase (POL), movement protein (MP), and three capsid proteins (Vp25, Vp20, and Vp24). (B) N. benthamiana plants were inoculated with empty vector (Mock), pCALSR1 + pCALSR2-XSB (ALSV), and pCALSR1 + pCALSR2-XSB:NbPDS (ALSV:PDS) by agroinoculation. Infection with ALSV (pCALSR1 + pCALSR2-XSB) is symptomless and infection with ALSV:PDS (pCALSR1 + pCALSR2-XSB:NbPDS) causes photobleaching of leaves by 14 days post-inoculation.

\section{AGROINOCULATION}

For agroinoculation, pCALSR1- and pCALSR2-based vectors were transformed into $R$. radiobacter strains GV3101::pMP90 and C58C1, respectively. The binary plasmids pBIN3, pBIN61:HCPro, pBE2113:2b, pBIN3:P19, pBE2113:50K, and pBIN3:AC2 were transformed into $R$. radiobacter strain $\mathrm{C} 58 \mathrm{C} 1$ ( $\mathrm{pCH} 32$ ).

Agroinoculation was carried out as described by Kon et al. (2009) with slight modifications. $R$. radiobacter strains were grown in $\mathrm{LB}$ medium containing the appropriate antibiotics at $30^{\circ} \mathrm{C}$. Cells were pelleted and resuspended in agroinduction buffer $(10 \mathrm{mM}$ MES, pH 5.7, $10 \mathrm{mM} \mathrm{MgCl}_{2}$, and $200 \mu \mathrm{M}$ acetosyringone) to a final OD $600=1.0$. The cell suspension was incubated for $3 \mathrm{~h}$ and then infiltrated onto the abaxial leaf surface of $N$. benthamiana and petunia (3-5 leaf stages) using a $1 \mathrm{ml}$ syringe without a needle.

\section{INDUCTION OF TGS}

The CaMV 35 S promoter (nt -343 to -32 ) was amplified by PCR with primers P5/P6 (Table 1), and then cloned into the
pGEM-T Easy vector and sequenced. Then the CaMV 35 S promoter (nt -343 to -32 ) was cloned into $M l u$ I/Nco I-digested pCALSR2-XSB/MN to generate pCALSR2-XSB/MN:35S. The $2 b$ and $P 19$ genes were respectively, amplified from pBE2113:2b and pBIN3:P19 using the primer pairs $\mathrm{P} 7 / \mathrm{P} 8$ and $\mathrm{P} 9 / \mathrm{P} 10$ (Table 1). PCR products were cloned into the pGEM-T Easy vector (Promega) and sequenced to confirm the absence of sequencing errors in the cloned genes in the constructs. Each suppressor gene was cloned between the Xho I and Bam HI sites of pCALSR2-XSB/MN:35S.

Total genomic DNA was extracted from petunia cv. Red Star, and the CHS-A1 promoter of Red Star petunia (nt 447 to -52 , Morita etal., 2012) was amplified by PCR with primers P11/P12 (Table 1). The amplified PCR product of CHSA1 promoter gene was cloned into the pGEM-T Easy vector and sequenced. A fragment of the $C H S-A 1$ promoter construct was cloned into $M l u$ I/Nco I-digested pCALSR2-XSB/MN to produce pCALSR2-XSB/MN:CHS-Apro. The cloned DNA was transformed into $R$. radiobacter strain $\mathrm{C} 58 \mathrm{C} 1$ and $N$. benthamiana 
Table 1 | Primer pairs used for PCR.

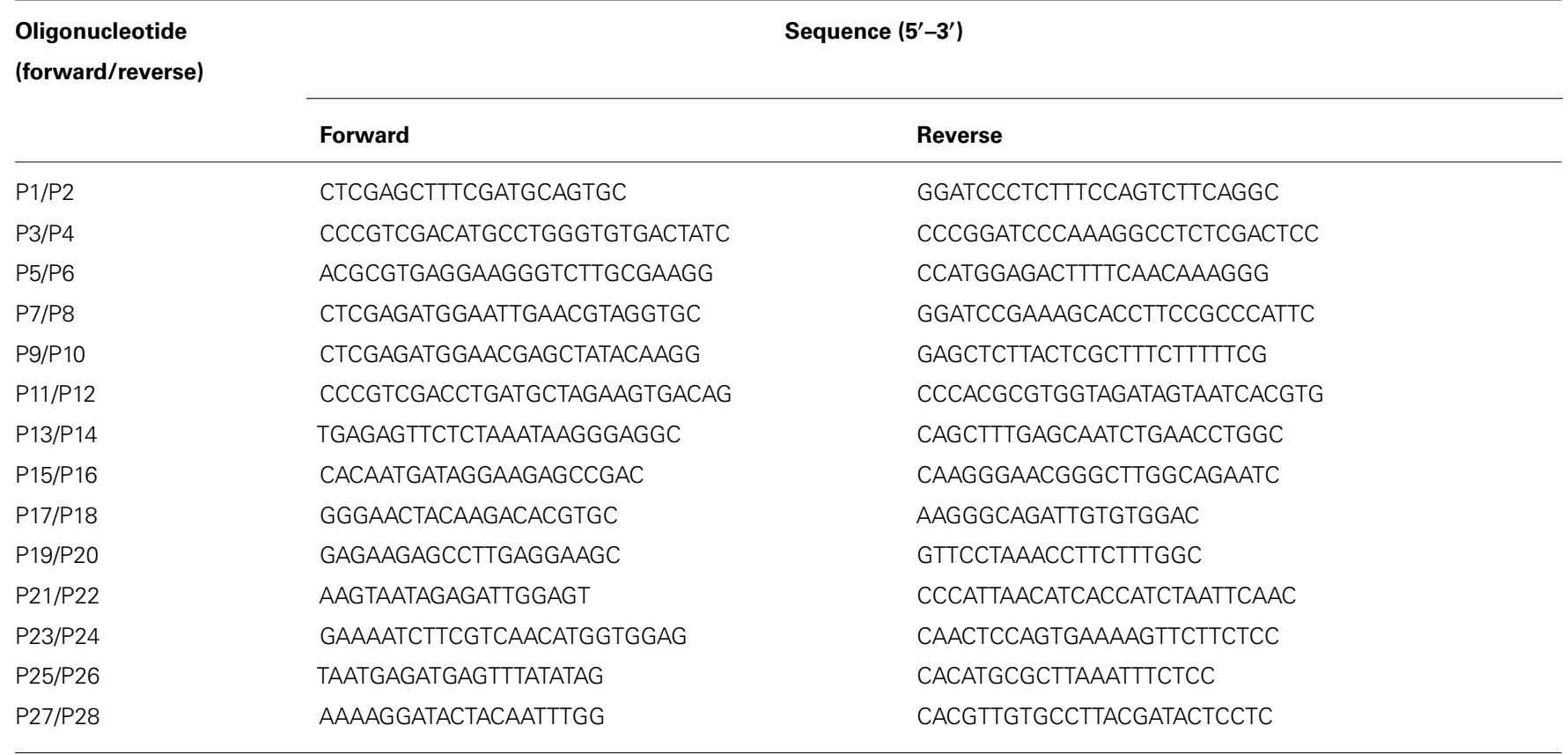

line 16c plants (kindly provided by Professor David Baulcombe) or agroinoculated into petunia plants as described above.

\section{RNA ANALYSIS}

Total RNA was extracted from agroinoculated plants using an RNeasy Plant Mini Kit (Qiagen) and then treated with DNase I. For RT-PCR, first-strand cDNA was synthesized by M-MuLV reverse transcriptase (New England Biolabs) with random 6mers. RT-PCR was carried out with ALSV RNA2 specific primers (P13/P14), 26S rRNA specific primers (P15/P16), GFP specific primers (P17/P18), or $C H S-A$ specific primers (P19/P20; Table 1). Isolation of low molecular weight RNA and detection of siRNAs by northern blotting were as described by Yaegashi etal. (2007). The membranes were hybridized with digoxigenin (DIG)-labeled RNA probes for the CaMV 35 S promoter sequence (nt -343 to -32 ) and the $C H S-A 1$ promoter gene (nt -447 to -52 ) of petunia cv. Red Star. siRNAs were immunodetected with anti-DIG Fab fragments coupled to alkaline phosphatase using an ImageQuant LAS4000 imager (GE Healthcare).

\section{BISULFITE SEQUENCING AND DNA METHYLATION ANALYSIS}

Total genomic DNA was extracted from leaves using a DNeasy plant mini kit (Qiagen). For bisulfite sequencing, $1 \mu \mathrm{g}$ of genomic DNA was treated with bisulfite using an EpiTect bisulfite kit (Qiagen) with slight modifications. The bisulfite-treated DNA was purified, and then the targeted regions were PCR-amplified. All primers for bisulfite sequencing analysis are listed in Table 1. For the CaMV 35S promoter in N. benthamiana line 16c plants, the target DNA region was amplified by a first round of PCR with primers $\mathrm{P} 21 / \mathrm{P} 22$. The product from the first reaction was used for a second round of PCR with primers P23/P24. For the
$C H S$ - $A$ promoter in petunia plants, the target DNA regions, $C H S$ $A 1$ and $C H S-A 2$, were amplified by a first round of PCR with primers P25/P26. The product from the first reaction was used for a second round of PCR with primers P27/P28. To determine whether the cytosine from the unmethylated DNA was converted completely to uracil by bisulfite treatment, unmethylated plasmid DNA containing the CaMV 35S promoter sequence was mixed with total plant genomic DNA, and then analyzed using bisulfite sequencing. The unmethylated plasmid DNA was amplified by PCR after treating with bisulfite and the cloned DNA showed conversion of cytosines to uracils (thymidines). The PCR products were cloned into the pGEM-T Easy vector and at least 10 clones per each position were sequenced. The sequences were aligned and analyzed by Kismeth software (Gruntman et al., 2008).

\section{RESULTS}

\section{CONSTRUCTION OF ALSV-BASED VECTORS IN A BINARY PLASMID AND AGROINOCULATION}

Li etal. (2004) reported the construction of ALSV RNA1 and RNA2 vectors in a pUC-based expression cassette vector (pEALSR1 and pEALSR2L5R5). In this study, the cDNAs of ALSV RNA1 in pEALSR1 and ALSV RNA2 in pEALSR2L5R5 were inserted into binary vector pCAMBIA1300, resulting in PCALSR1 and PCALSR2-XSB, respectively (Figure 1A). When pCALSR1 and pCALSR2-XSB were agroinoculated together into $N$. benthamiana plants, they had a $100 \%$ infection rate (Table 2). Infected N. benthamiana plants did not show any obvious symptoms (Figure 1B; ALSV), as described before (Yamagishi et al., 2011).

Apple latent spherical virus-RNA vectors, which have cloning sites in the $3^{\prime}$ non-coding region of RNA2, were developed in this study (Figure 1A). When ALSV RNA-based vectors 
Table 2 | Effects of viral silencing suppressors on infectivity of apple latent spherical virus vectors following agroinoculation of Nicotiana benthamiana.

\begin{tabular}{lll}
\hline Inoculum & Viral suppressor & Infectivity \\
\hline pCALSR1 + pCALSR2-XSB & - & $10 / 10$ \\
pCALSR1 + pCALSR2-XSB/MN & - & $2 / 10$ \\
pCALSR1 + pCALSR2-XSB/MS & - & $2 / 10$ \\
pCALSR1 + pCALSR2-XSB/NS & - & $2 / 10$ \\
pCALSR1 + pCALSR2-XSB/SM & - & $2 / 10$ \\
pCALSR1 + pCALSR2-XSB/MN + & $2 b$ & $10 / 10$ \\
pBE2113:2b & & \\
pCALSR1 + pCALSR2-XSB/NS + & AC2 & $10 / 10$ \\
pBIN3:AC2 & & \\
pCALSR1 + pCALSR2-XSB/MS + & P19 & $10 / 10$ \\
pBIN3:P19 & & \\
pCALSR1 + pCALSR2-XSB/SM + & $50 K P$ & $10 / 10$ \\
pBE2113:50K & & \\
pCALSR1 + pCALSR2-XSB/SM + & HC-Pro & $10 / 10$ \\
pBIN61:HC-Pro & &
\end{tabular}

${ }^{a}$ RNA silencing suppressors expressed from co-infiltrated plasmid DNAs. The symbols 2b, AC2, P19, 50KP, and HC-Pro are suppressor proteins of CMV, ToMHV, TBSV, ACLSV, and PVY, respectively.

${ }^{\mathrm{b}}$ Number of infected plants/inoculated plants based on the detection of viral RNA by RT-PCR. Sum of two independent experiments.

having different restriction enzyme sites (pCALSR2-XSB/MN, pCALSR2-XSB/MS, pCALSR2-XSB/NS, and pCALSR2-XSB/SM) were co-agroinoculated with pCALSR1 into $N$. benthamiana, $20 \%$ of plants were infected with each ALSV RNA2 construct (Table 2). ALSV Vp24 protein is a weak suppressor of local RNA silencing but a potential suppressor of systemic RNA silencing in plants (Yaegashi et al., 2007). The modified ALSV RNA2-based vectors may cause reduced accumulation of viral RNA during the initial infection stage, and be affected by RNA silencing in plants inoculated with them. To improve the efficiency of infection by agroinoculation of the ALSV-based vector system, RNA silencing suppressors were co-expressed in $\mathrm{N}$. benthamiana plants. When pCALSR1 and pCALSR2-XSB/MN were co-agroinoculated with a DNA construct transiently expressing a viral RNA silencing suppressor (pBE2113:2b, pBIN3:AC2, pBIN3:P19, pBE2113:50K, or pBIN61:HC-Pro) into N. benthamiana plants, all the inoculated plants were infected, indicating that co-expression with RNA silencing suppressors can increase the infection efficiency of pCALSR2-XSB/MN by agroinoculation from 20 to $100 \%$ (Table 2). The presence of viral RNAs was detected by RT-PCR using specific primers, and when upper leaves from plants systemically infected with virus were homogenized, subsequent sap-inoculation led to successful infections. When pCALSR2-XSB:NbPDS containing a PDS gene fragment of $N$. benthamiana was inoculated with PCALSR1, photobleaching of upper leaves appeared 14 days after inoculation (Figure 1B; ALSV:PDS), indicating that virus derived from ALSV RNA1 and modified RNA2-based vectors had systemically infected the plant.
These results showed that most plant RNA silencing suppressors increase ALSV vector infectivity. Furthermore, agroinoculated $N$. benthamiana plants provided a source of viral inoculum for subsequent inoculation of other experimental host plants.

\section{INDUCTION OF VITGS OF THE CAMV 35S TRANSGENE PROMOTER BY THE ALSV VECTOR}

To investigate whether the ALSV vector induces DNA methylation, DNA methylation patterns in the CaMV 35 S promoter region of transgenic $N$. benthamiana line 16c plants expressing GFP were analyzed. The CaMV $35 \mathrm{~S}$ promoter (nt -343 to -32) was cloned into the ALSV RNA2-based vector (pCALSR2$\mathrm{XSB} / \mathrm{MN}$ ) to produce pCALSR2-XSB/MN:35S (Figure 2A), and then recombinant ALSV (ALSV:35S) was inoculated into line $16 \mathrm{c}$ plants expressing GFP under the control of the CaMV 35S promoter. When a wild-type ALSV and ALSV:35S were inoculated into line $16 \mathrm{c}$ plants, mock- or ALSV-inoculated line $16 \mathrm{c}$

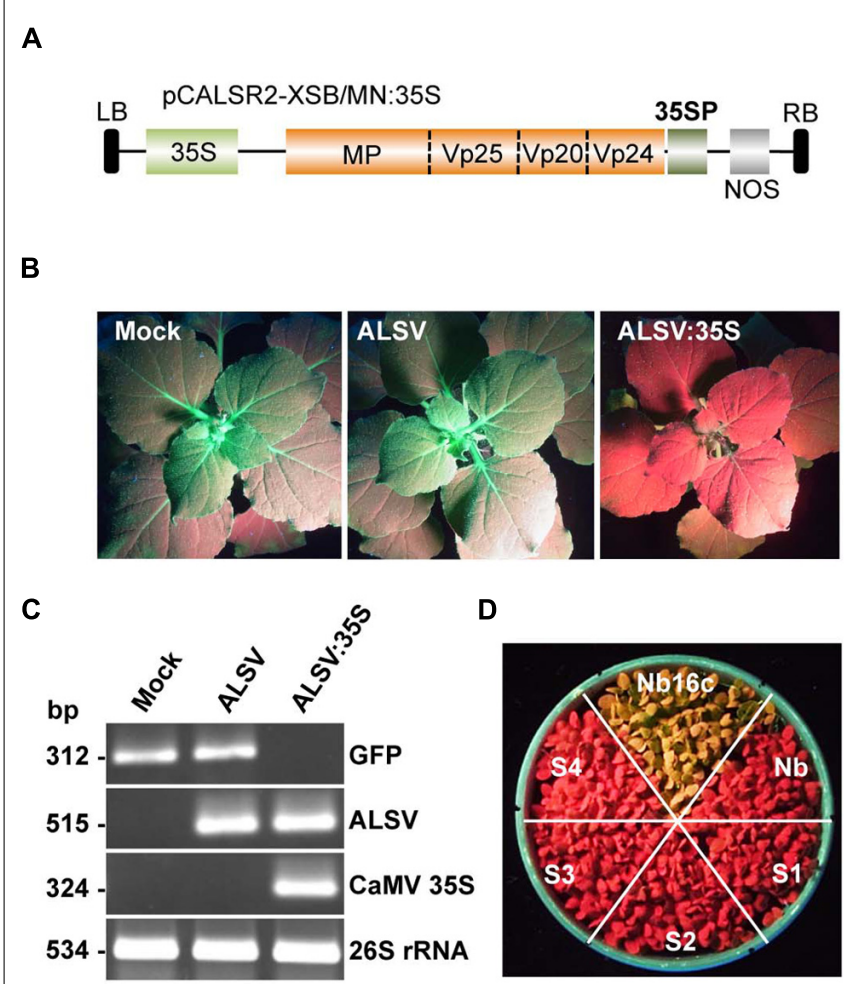

FIGURE 2 | Induction of TGS by the ALSV vector and its inheritance. (A) Schematic representation of ALSV vector containing part of the $35 \mathrm{~S}$ promoter (35SP; pCALSR2-XSB/MN:35S). (B) N. benthamiana line 16c plants expressing GFP and mock-agroinoculated (Mock), or agroinoculated with pCALSR1 + pCALSR2-XSB (ALSV) or pCALSR1 + pCALSR2-XSB/MN:35S (ALSV:35S). Plants were photographed under UV illumination with a yellow filter. The red fluorescence reveals that TGS of GFP occurred in ALSV:35S-infected tissues. (C) Analysis of mRNAs in agroinoculated $\mathrm{N}$. benthamiana line $16 \mathrm{c}$ plants by RT-PCR. Total plant RNA was extracted from plants agroinoculated with empty vector (lane 1), ALSV (lane 2), or ALSV:35S (lane 3). (D) The silenced generations 1-4 (S1-S4) seedlings derived from ALSV:35S infected plants (SO), showing disappearance of GFP fluorescence. The progeny of non-transgenic $N$. benthamiana plants $(\mathrm{Nb})$ and mock-inoculated $\mathrm{N}$. benthamiana line $16 \mathrm{c}$ (Nb16c) are shown as controls. 
plants maintained green fluorescence whereas line $16 \mathrm{c}$ plants inoculated with ALSV:35S showed a loss of green fluorescence by 3 weeks post-inoculation (Figure 2B). RT-PCR failed to detect any GFP mRNA from line 16c plants inoculated with ALSV:35S (Figure 2C). These results suggest that the reduction in GFP mRNA is the result of VITGS due to ALSV:35S infection.

Seeds from line 16c plants showing a loss of GFP fluorescence following inoculation with ALSV:35S were harvested to investigate whether VITGS induced by the ALSV vector is inheritable by analyzing the progeny [named silenced generation 1 (S1) plants] from ALSV:35S-infected plants [silenced generation 0 (S0) plants]. None of the S1 progeny obtained from S0 plants showed GFP fluorescence, as shown by the red color in Figure 2D. The progeny of the S2-S4 generations, which were free from ALSV infection, also did not show GFP fluorescence (Figure 2D). These results indicate that the ALSV vector carrying a plant promoter gene induces DNA methylation of genomic DNA and transcriptionally suppresses the target gene, and that VITGS can be inherited by the next generation.

Next, the DNA methylation patterns of the CaMV $35 \mathrm{~S}$ promoter in ALSV:35S-infected line 16c plants were analyzed by bisulfite sequencing. The target region ( $\mathrm{nt}-343$ to -32 ) of the CaMV 35S promoter contains $12 \mathrm{CG}, 7 \mathrm{CHG}$, and $62 \mathrm{CHH}$ sites. The genomic DNA was treated with bisulfite. In mockinoculated plants, the cytosine residue at the CHG site was never methylated and only a small number of cytosine residues at the CG and $\mathrm{CHH}$ sites were methylated $(2.1$ and $1.8 \%$, respectively; Figures 3A,B). In contrast, in plants agroinoculated with ALSV:35S, the CG and CHG sites were highly methylated (91.7 and $95.6 \%$, respectively). The cytosine residue of the $\mathrm{CHH}$ site was also methylated in $35.3 \%$ of plants (Figures $3 \mathbf{A , B}$ ). In S1 progeny from ALSV:35S-infected line 16c plants (S0 plants), cytosine methylation patterns were highly maintained at the CG and CHG sites, in 90.6 and $87.5 \%$ of plants, respectively (Figures 3A,B). On the other hand, cytosine methylation at $\mathrm{CHH}$ sites of S1 plants was $5.1 \%$, low compared with S0 plants $(35.3 \%$; Figures 3A,B).

The region of the CaMV $35 \mathrm{~S}$ promoter ( $\mathrm{nt}-343$ to -32 , corresponding to defining the transcriptional start site as zero) has a transcriptional regulation domain, which contains two tandem repeats (TGACG, nt -82 to -78 and -70 to -66 ), named the activation sequence 1 element (Benfey and Chua, 1990). The activation sequence factor binds to the activation sequence 1 element (Kanazawa et al., 2007). Bisulfite sequencing revealed that cytosine methylation occurred $100 \%$ at the CG site in the activation sequence 1 element of the CaMV $35 \mathrm{~S}$ promoter both in ALSV:35S-infected S0 plants and in S1 plants (Figures 3A,B). The DNA methylation patterns of the CaMV 35S promoter in the progeny (S2-S4) were analyzed by bisulfite sequencing. All plants of the S1-S4 generations showed cytosine methylation patterns that were highly maintained at the CG $(>89 \%)$ and CHG (>94\%) sites (Figures 3A,B). Thus, an ALSV-based VITGS vector could induce both DNA methylation and TGS in the homologous promoter region, suggesting that cytosine methylation at $\mathrm{CG}$ and $\mathrm{CHG}$ sites is highly inherited by subsequent generations.

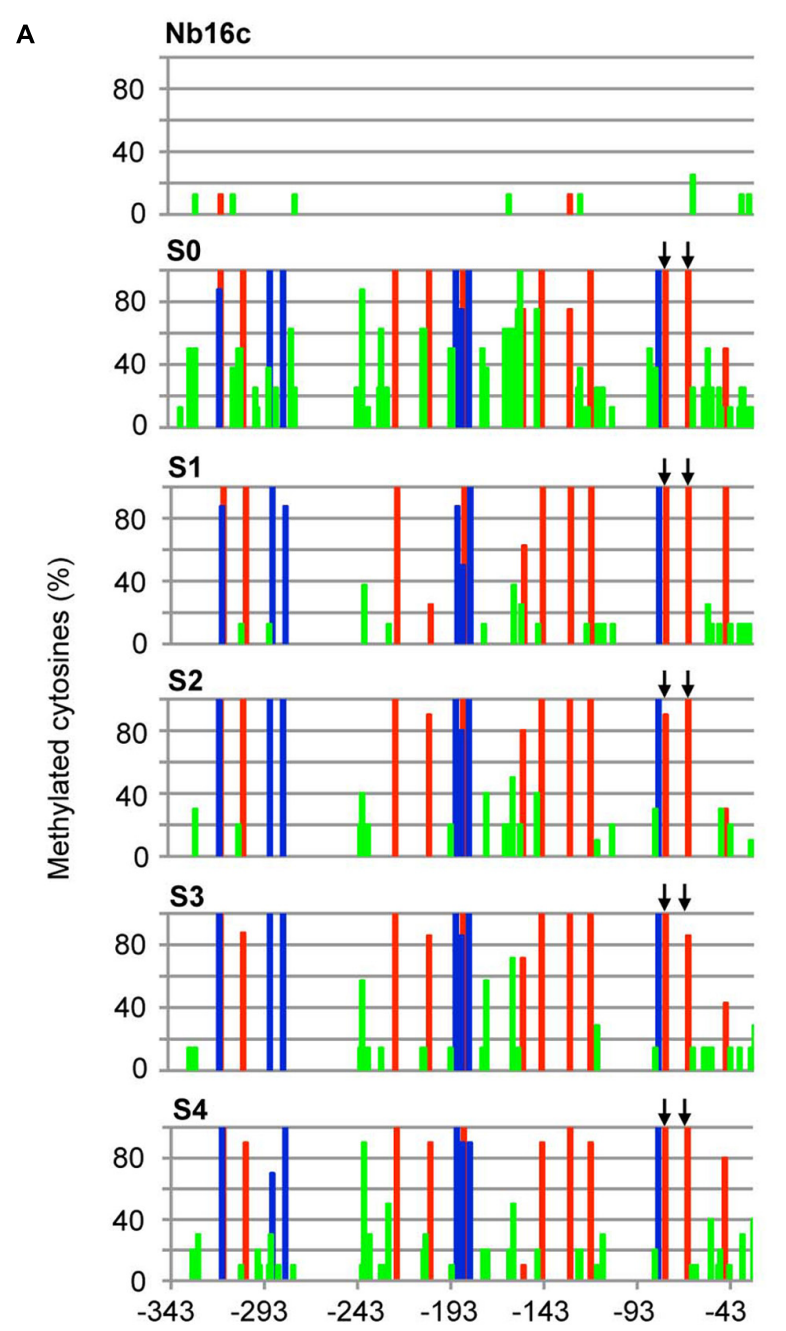

B

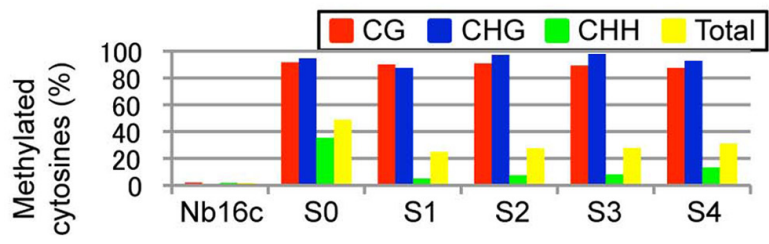

FIGURE 3 | Cytosine methylation status of the CaMV 35 S promoter region. (A) Total plant DNA from mock-agroinoculated $N$. benthamiana line $16 \mathrm{c}$ plants (Nb16c), Nb16c plants infected with ALSV:35S (S0), and progeny from four generations of ALSV:35S-infected Nb16c plants (S1, S2, S3, and S4) were treated with bisulfite. The arrows indicate elements within the domain of activation sequences. (B) Summary of bisulfite sequencing analysis. The red, blue, green, and yellow bars respectively represent the percentage of cytosines methylated at the $\mathrm{CG}, \mathrm{CHG}, \mathrm{CHH}$, and all contexts.

\section{RNA SILENCING SUPPRESSOR INHIBITS ALSV-MEDIATED VITGS IN PLANTS}

A recent study showed that CMV-encoded $2 \mathrm{~b}$ protein promotes efficient epigenetic modification through the transport of the 2b-siRNA complex to the nucleus (Kanazawa et al., 2011). To test whether the $2 \mathrm{~b}$ protein could more effectively promote 
ALSV-mediated VITGS, an ALSV vector was constructed to express the $2 \mathrm{~b}$ protein as well as to silence the CaMV $35 \mathrm{~S}$ promoter in $N$. benthamiana line $16 \mathrm{c}$ plants. The $2 \mathrm{~b}$ protein was cloned into multiple cloning sites in the coding region of a recombinant ALSV RNA2 vector carrying the CaMV 35S promoter sequence (pCALSR2-XSB/MN:35S) to produce pCALSR2$\mathrm{XSB}: 2 \mathrm{~b} / \mathrm{MN}: 35 \mathrm{~S}$ (ALSV:2b/35S; Figure 4A). When the resulting ALSV:2b/35S was used for inoculation of line $16 \mathrm{c}$ plants, all plants infected with ALSV:2b/35S retained GFP fluorescence in the upper leaves (Figure 4B). A similar result was obtained in line

A pCALSR2-XSB:2b/MN:35S PCALSR2-XSB:P19/MN:35S

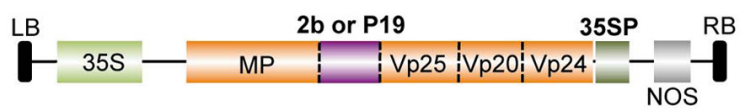

B
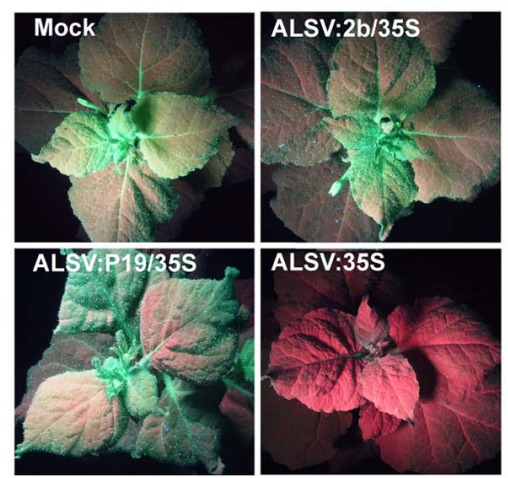

C

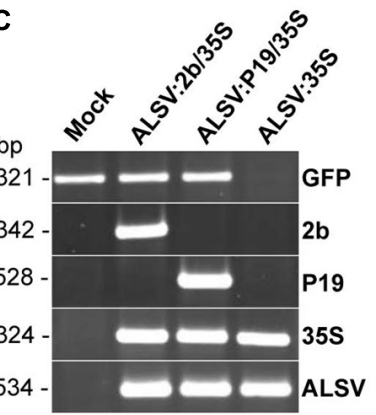

D

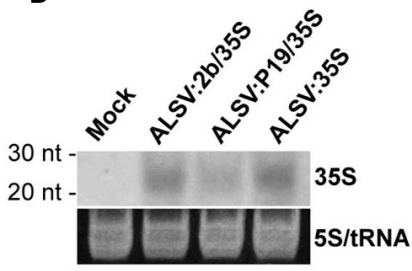

FIGURE 4 | Induction of TGS by ALSV vectors carrying an RNA silencing suppressor. (A) Schematic representation of ALSV vectors expressing a plant virus suppressor (2b or P19; pCALSR2-XSB:2b/MN:35S or pCALSR2-XSB:P19/MN:35S). (B) N. benthamiana line 16c plants expressing GFP and mock-agroinoculated (Mock), agroinoculated with pCALSR1 + pCALSR2-XSB:2b/MN:35S (ALSV:2b/35S), pCALSR1 + pCALSR2-XSB:P19/MN:35S (ALSV:P19/35S), or pCALSR1 + pCALSR2-XSB/MN:35S (ALSV:35S). Plants were photographed under UV illumination with a yellow filter. The red fluorescence reveals that TGS of GFP occurred in ALSV:35S infected tissues. (C) Analysis of mRNAs in agroinoculated $N$. benthamiana line $16 \mathrm{c}$ plants by RT-PCR. Total plant RNA was extracted from plants agroinoculated with empty vector (Mock), ALSV:2b/35S, ALSV:P19/35S, or ALSV:35S. (D) Northern blot analysis of siRNAs corresponding to the CaMV 35 S promoter gene. Total RNA was extracted from plants agroinoculated with empty vector (Mock),

ALSV:2b/35S, ALSV:P19/35S or ALSV:35S, and hybridized with a sense DIG-labeled RNA probe of the CaMV 35 S promoter. The 5S rRNA and tRNA were stained by ethidium bromide and are shown as a loading control. 16c plants infected with ALSV:P19/35S (Figure 4B). In contrast, GFP fluorescence disappeared in line $16 \mathrm{c}$ plants infected with ALSV:35S (Figure 4B). RT-PCR showed that GFP mRNA could be detected from line 16c plants infected with either ALSV:2b/35S or ALSV:P19/35S, but not from line 16c plants infected with ALSV:35S (Figure 4C). Northern blotting showed that CaMV 35S-derived siRNAs were detected from the upper leaves of plants infected with ALSV carrying CaMV 35S fragment vectors (Figure 4D). These results suggest that viral silencing suppressors (2b and P19) inhibit ALSV-mediated VITGS in plants.

\section{INDUCTION OF ALSV-MEDIATED VITGS OF AN ENDOGENOUS GENE IN PLANTS}

To investigate whether the ALSV-VITGS vector can induce DNA methylation in an endogenous plant gene, the DNA methylation patterns were analyzed in an endogenous gene promoter in petunia plants. The natural bicolor trait of petunia cv. Red Star is caused by post-TGS of the two CHS-A gene copies (Figure 5A; Morita et al., 2012). The promoter of the chalcone synthase-A (CHS-A) gene, which encodes an anthocyanin biosynthetic enzyme, was cloned into the ALSV RNA2-based vector to produce pCALSR2-XSB/MS:CHS-Apro (Figure 5B), and then the recombinant ALSV vector (ALSV:CHS-Apro) was agroinoculated into petunia plants. When petunia plants were infected with wild-type ALSV, the flower color pattern did not change, as also observed with mock-agroinoculated plants (Figure 5C). However, petunia plants infected with ALSV:CHS-Apro showed white petal patterns (Figure 5C). A similar result was obtained for petunia plants infected with ALSV vector carrying a partial fragment of the $C H S$ - $A$ gene coding region (ALSV:CHS-A; Figures 5B,C).

Total RNA was extracted from the white tissue of petunia flowers and analyzed by RT-PCR. CHS-A mRNA was reduced in plants infected with ALSV:CHS-A and ALSV:CHS-Apro, in contrast to plants treated by mock-agroinoculation or infected with ALSV (Figure 5D). The level of $C H S$ - $A$ specific siRNA in infected plants was assayed by northern blotting. $C H S$ - $A$ promoter specific siRNA was detected from plants infected with ALSV:CHS-Apro, but not from plants inoculated with a mock treatment or with ALSV or ALSV:CHS-A (Figure 5E).

Next, the DNA methylation patterns of the CHS-A promoter from white tissue of flowers infected with ALSV:CHS-Apro were analyzed by bisulfite sequencing. Petunia cv. Red Star plants have two copies of the CHS-A gene (designated CHS-A1 and CHS-A2; Morita et al., 2012; Figure 5A). The $C H S-A$ promoter region (nt -447 to -53 , corresponding to the transcriptional start site at 1 for the CHS-A1 promoter and nt -269 to -52 for the CHS-A2 promoter) has potential transcriptional regulation domains (e.g., an anther box, CACGTG motifs, and TACPyAT boxes; van der Meer et al., 1990). In mock-agroinoculated plants, no cytosine residues at $\mathrm{CG}, \mathrm{CHG}$, and $\mathrm{CHH}$ sites were methylated except for a 179-bp insertion fragment within the promoter (Figure 6A). The $C H S-A 1$ promoter sequence has a transposon-like insertion fragment (Morita etal., 2012). Bisulfite sequencing showed that the 179-bp insertion fragment region of the CHS-A1 promoter in mock-agroinoculated plants was highly methylated (Figure 6A). The cytosine residues at the CG, $\mathrm{CHG}$ and $\mathrm{CHH}$ sites including the 


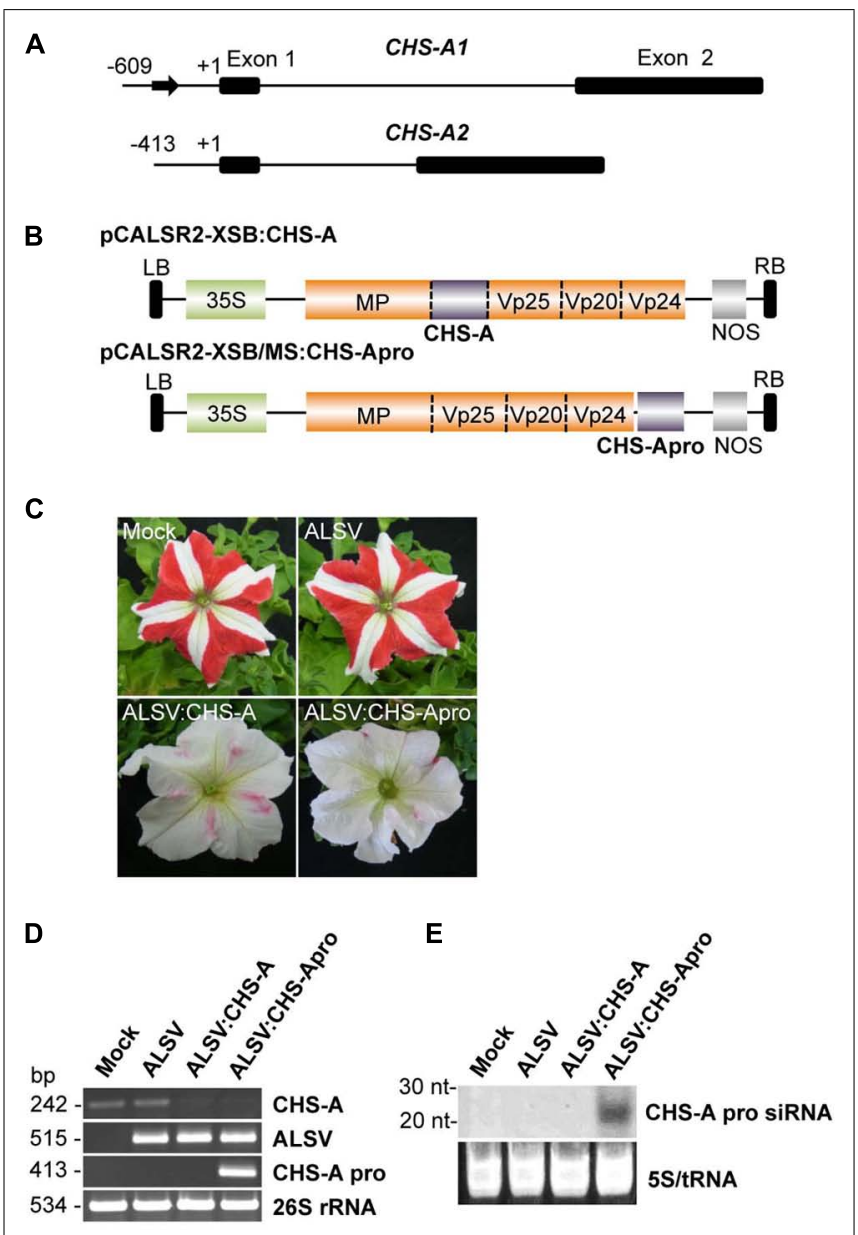

FIGURE 5 | Induction of TGS by ALSV vector carrying an endogenous petunia gene. (A) The structure of the CHS-A genes. The sequence described by Morita etal. (2012) is in the DDBJ/EMBL/GenBank database under the accession number $A B 678719$. Arrow indicates a transposon-like 179-bp insertion fragment in the CHS-A1 promoter region. Nucleotide numbering is relative to the transcription start site. (B) Schematic representation of ALSV vectors for VIGS (pCALSR2-XSB:CHS-A) and VITGS (pCALSR2-XSB/MS:CHS-Apro) of CHS-A genes. (C) Petunia flower color phenotypes. Petunia cv. Red Star plants agroinoculated with empty vector (Mock), pCALSR1 + pCALSR2-XSB (ALSV), pCALSR1 + pCALSR2-XSB:CHS-A (ALSV:CHS-A), or pCALSR1 + pCALSR2-XSB/ MS:CHS-Apro (ALSV:CHS-Apro). (D) Analysis of mRNAs in agroinoculated petunia plants by RT-PCR. Total RNA was extracted from plants agroinoculated with empty vector (Mock), ALSV, ALSV:CHS-A, and ALSV:CHS-Apro. The 26S rRNA was amplified by PCR as an internal control. (E) Northern blot analysis of siRNAs corresponding to the CHS-A promoter gene. Total RNA was extracted from plants agroinoculated with empty vector (Mock), ALSV, ALSV:CHS-A, or ALSV:CHS-Apro and hybridized with a sense DIG-labeled RNA probe for the CHS-A promoter. The 5S rRNA and tRNA were stained by ethidium bromide and are shown as a loading control.

179-bp insertion fragment region, were respectively methylated 31.9, 33.3, and 30.4\% in mock-agroinoculated plants (Figure 6B). In contrast, petunia plants infected with ALSV:CHS-Apro showed methylation at the CG, CHG, and CHG sites including the 179bp insertion fragment region in $65.8,70$, and $47.3 \%$, respectively (Figures 6A,B). The percentage of methylation at the $\mathrm{CHH}$ sites in the $C H S-A 1$ promoter in infected plants was higher than that in the region of the CaMV $35 \mathrm{~S}$ promoter of line $16 \mathrm{C}$ plants (Figure 3). This may be due to the presence of transposon-like sequences in $C H S$ - $A 1$ which were highly methylated in $\mathrm{CHH}$ sites in mock-inoculated plants (Figure 6B). Cytosine methylation of the $C H S-A 2$ promoter gene was also found in plants infected with ALSV:CHS-Apro, and the CG, $\mathrm{CHG}$, and $\mathrm{CHH}$ sites were methylated 30.3, 77.2, and 25.4\%, respectively (Figures 6C,D). In mock-agroinoculated plants, a small number of cytosine residues of the CHS-A2 promoter gene were methylated at CHG $(12.5 \%)$ and $\mathrm{CHH}(0.4 \%)$ sites (Figures 6C,D). These results suggest that transcriptional suppression occurs in plants infected with ALSV vectors carrying a promoter sequence and that the VITGSderived siRNA induces DNA methylation of the endogenous plant gene.

\section{DISCUSSION}

The VIGS system has been used for functional analysis of genes in diverse plant species. Numerous plant viral vectors including DNA and RNA viruses have been developed (Becker and Lange, 2010). In addition, an advantage of a plant viral-based VIGS system is the ability to introduce viral vectors simply into plants by agroinoculation. Potato virus X (PVX)-based vectors have been developed for gene expression and RNA silencing studies (Baulcombe et al., 1995; Jones et al., 1999), but a PVX-based vector has a limited host range. On the other hand, a tobacco rattle virus (TRV)-based vector has been developed and used for a number of VIGS studies (Liu et al., 2002; Brigneti et al., 2004; Burch-Smith et al., 2004). The advantages of the TRV-based vector are easy induction of RNA silencing via a simple agroinoculation method and large-scale screening for functional gene analysis. The ALSV-based vector constructed in our laboratory has several advantages such as more extensive spreading throughout plant tissue, including growing regions, without any obvious symptoms and the ability to infect economically important crops such as solanaceous plants (tobacco, tomato, potato etc.), legumes (soybean, broad bean, azuki bean, pea, etc.), cucurbits (cucumber, melon, squash, luffa etc.; Igarashi et al., 2009), and fruit trees including apple, pear, peach, plum, citrus, and grapevine (Sasaki et al., 2011, unpublished data). However, the infectivity of ALSV vectors in a pUC-based expression cassette vector (pEALSR1 and pEALSR2L5R5; Li et al., 2004) was not high enough for direct infection of most plants, and the vectors needed to be first inoculated into C. quinoa plants by a mechanical inoculation method (infection rate, $0 \sim 50 \%$ ) for virus propagation, followed by inoculation of virus to the target plants. In this paper we developed an agroinoculation system for the ALSV vector. This system, combined with co-expression of a virus silencing suppressor, increased the efficiency of ALSV-based vector infection in N. benthamiana plants.

RNA-mediated TGS could target to a gene promoter in genomic DNA via small RNAs (Pontes et al., 2006; He et al., 2009). A TRV vector has been used for inducing DNA methylation on the promoter region of a transgene (Jones et al., 2001). A CMV vector also effectively induces both transgene and endogenous gene promoter TGS in plant genomic DNA (Kanazawa etal., 2011). These viruses generate dsRNA intermediates, which are targeted by the host defense RNA silencing pathway to produce small RNAs. Therefore, infection of plant viral vectors carrying 


\section{A}

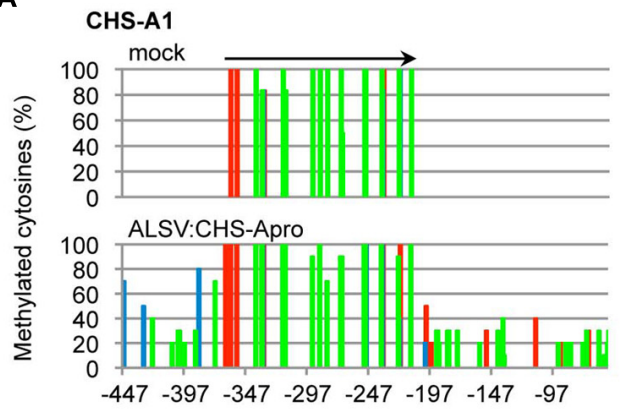

C

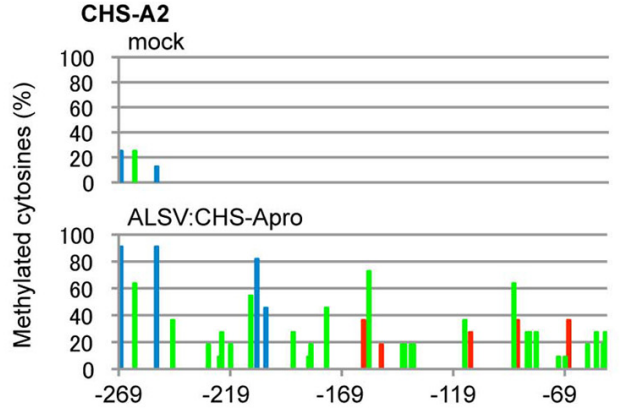

B

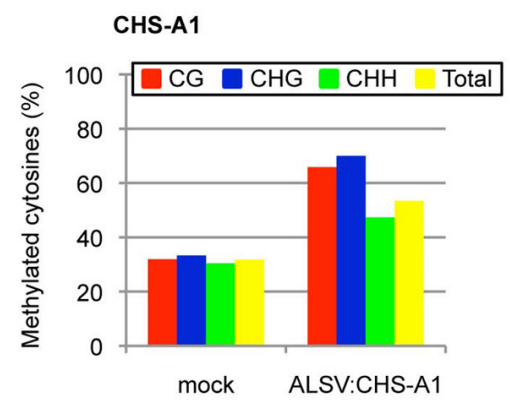

D

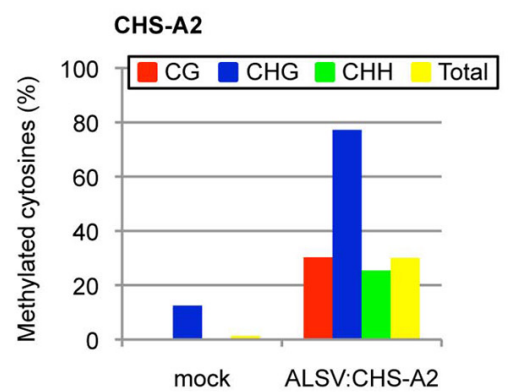

FIGURE 6 | Cytosine methylation status of the promoter of $\mathrm{CHS}-\mathrm{A1}$ and $\boldsymbol{C H S}$-A2. Total DNA was extracted from mock-agroinoculated petunia plants and from plants infected with ALSV:CHS-Apro and treated with bisulfite. The red, blue, and green bars respectively represent the percentage of cytosines methylated at the $\mathrm{CG}, \mathrm{CHG}$, and $\mathrm{CHH}$ sites.
Arrow indicates a transposon-like 179-bp insertion fragment in the CHS-A1 promoter region. (A) Cytosine methylation of CHS-A1. (B) Summary of bisulfite sequencing analysis of the $C H S-A 1$ promoter gene. (C) Cytosine methylation of CHS-A2. (D) Summary of bisulfite sequencing analysis of the $C H S-A 2$ promoter gene. a promoter gene can induce TGS at homologous regions in the genome. The results in this paper demonstrated that an ALSVbased vector carrying a promoter gene effectively induces TGS on the homologous genomic promoter region. This suggests that ALSV produces virus-derived small RNAs during viral replication, and that the small RNAs themselves may directly move to the nucleus and induce TGS. A recent study has shown that endogenous mobile small RNAs from source cells direct epigenetic modifications of the genome in recipient cells (Molnar et al., 2010).

Cucumber mosaic virus $2 \mathrm{~b}$ protein binds to small RNAs and the 2b-small RNA complex moves to the nucleus (Kanazawa etal., 2011). Thus, the small RNA-binding activity of the $2 b$ protein is thought to be important for induction of TGS in the nucleus (Kanazawa etal., 2011). In this paper, we showed that viral silencing suppressors (tomato bushy stunt virus p19 and CMV 2b) expressed in an ALSV vector block VITGS in N. benthamiana plants (Figure 4). Since an ALSV-based vector can effectively induce TGS, a nuclear targeting protein such as $2 \mathrm{~b}$ may not be required for TGS induction in plants infected with ALSV vectors. At present, we have no idea on this discrepancy between CMV and ALSV systems. As the suppressor activity of the $2 \mathrm{~b}$ protein increased the infection efficiency of ALSV vectors by agroinoculation (Table 2), probably due to the inhibition of RNA silencing induced by ALSV infection, the expression of $2 \mathrm{~b}$ protein may also reduce the production of small-RNAs that act as inducers of TGS in infected $N$. bentamiana.

The ALSV-based vector system effectively induces DNA methylation in plants via VITGS. In animals, DNA methylation normally occurs at a CG site, whereas the cytosine at CG, CHG, and $\mathrm{CHH}$ sites can be methylated in plants. When ALSV:35S was introduced into GFP-expressing transgenic line $16 \mathrm{c}$ plants, the targeted CaMV $35 \mathrm{~S}$ promoter region was highly methylated at CG and CHG sites, and cytosine methylation was also found at the $\mathrm{CHH}$ site. In plants, RdDM is required for a dsRNA trigger and de novo DNA methylation can be induced by DRM methyltransferases at CG, $\mathrm{CHG}$, and $\mathrm{CHH}$ sites (Cokus et al., 2008). In this study, cytosine methylation at CG and CHG sites was highly inherited and resulted in down-regulation of gene transcription in S1 progeny (Figure 3). In plants, three methyltransferases (MET1, CMT3, and DRM2) have been identified (Cao et al., 2003; Chan et al., 2005). Two maintenance class methyltransferases, MET1 and CMT3, are required for cytosine methylation at $\mathrm{CG}$ and $\mathrm{CHG}$ sites, respectively (Henderson and Jacobsen, 2007). In S1 progeny, a high cytosine methylation status (>90\%) was found at CG and $\mathrm{CHH}$ sites, but not at $\mathrm{CHH}$ sites ( $\sim 5 \%)$ in contrast to $\mathrm{CHH}$ sites $(\sim 35 \%)$ in S0 plants (Figure 3). The cytosine methylation at CG and CHG sites in S1 plants may be maintained by the two maintenance class methyltransferases. Cytosine methylation at $\mathrm{CHH}$ sites in $\mathrm{S} 1$ plants resulted in de novo DNA methylation. This cytosine methylation at $\mathrm{CHH}$ sites may be controlled by a de novo class methyltransferase 
(e.g., DRM2) or maintained by maintenance class methyltransferases (e.g., MET1 and CMT3; Cao et al., 2003). Previous findings have shown that cytosine methylation at $\mathrm{CHH}$ sites at some loci in plants is controlled by CMT3 and DRM2 (Cao et al., 2003; Chan et al., 2005). Maintenance of the cytosine methylation at $\mathrm{CHH}$ sites in RdDM normally requires for the presence of a dsRNA trigger (Vaucheret, 2006; Zaratiegui et al., 2007). The current results suggest that asymmetric $\mathrm{CHH}$ methylation in $\mathrm{S} 0$ plants is inherited, and is maintained in S1 plants in the absence of dsRNA, because the recombinant ALSV vector carrying the promoter gene was not detected in S1 plants.

Here, transcriptional silencing of an endogenous gene by its native promoter was demonstrated after recombinant ALSVbased vector infection. The level of DNA methylation of an endogenous gene promoter (e.g., the $C H S$ - $A$ promoter) was also induced in wild-type petunia plants infected with ALSV:CHSApro (Figure 6). A de novo class DNA methyltransferase (i.e., DRM2 and its orthologs) controls the dsRNA-mediated RdDM pathway (Cao et al., 2003; Chan et al., 2005). The methylation status of $\mathrm{CG}, \mathrm{CHG}$, and $\mathrm{CHH}$ sequences may differ among plant species, or DNA methyltransferase activity may depend on plant developmental stage (Cao and Jacobsen, 2002; Cao et al., 2003). However, no studies on biochemical properties of DRM orthologs from petunia plants have been reported. It will be interesting to see whether the DRM protein expressed by the ALSV vector can increase DNA methylation frequency at target sites in plants.

This study demonstrated that agroinoculation of modified ALSV-based vectors and co-expression of RNA silencing suppressors from diverse plant viruses increased the efficiency of agroinoculation. In addition, the modified ALSV-based vector induced effective TGS of a promoter gene in plants. Thus, the ALSV-based VITGS system provides a useful tool for epigenetic modification and could be used as a technique for biotechnology-based plant breeding (Hartung and Schiemann, 2014).

\section{ACKNOWLEDGMENTS}

The authors would like to thank the late Professor Hiroshi Kamata for his helpful suggestions. They also thank Professor David Baulcombe for providing $N$. benthamiana line $16 \mathrm{c}$ and pBIN61:HC-Pro, Professor Masato Ikegami for providing binary vector pBIN3, and Professor Robert Gilbertson for providing pBIN3:AC2. This work was supported by the Programme for Promotion of Basic and Applied Researches for Innovations in Bio-oriented Industry from the Ministry of Agriculture, Forestry and Fisheries, Japan.

\section{REFERENCES}

Baulcombe, D. (2004). RNA silencing in plants. Nature 431, 356-363. doi: 10.1038 /nature 02874

Baulcombe, D. C., Chapman, S. N., and Santa Cruz, S. (1995). Jellyfish green fluorescent protein as a reporter for virus infections. Plant J. 7, 1045-1053. doi: 10.1046/j.1365-313X.1995.07061045.x

Becker, A., and Lange, M. (2010). VIGS - genomics goes functional. Trends Plant Sci. 15, 1-4. doi: 10.1016/j.tplants.2009.09.002

Benfey, P. N., and Chua, N.-H. (1990). The cauliflower mosaic virus 355 promoter: combinatorial regulation of transcription in plants. Science 250, 959-966. doi: 10.1126/science.250.4983.959
Brigneti, G., Martin-Hernandez, A. M., Jin, H. L., Chen, J., Baulcombe, D. C., Baker, B., et al. (2004). Virus-induced gene silencing in Solanum species. Plant J. 39, 264-272. doi: 10.1111/j.1365-313X.2004.02122.x

Burch-Smith, T. M., Anderson, J. C., Martin, G. B., and Dinesh-Kumar, S. P. (2004). Applications and advantages of virus-induced gene silencing for gene function studies in plants. Plant J. 39, 734-746. doi: 10.1111/j.1365-313X.2004. 02158.x

Cao, X., Aufsatz, W., Zilberman, D., Mette, M. F., Huang, M. S., Matzke, M., et al. (2003). Role of the DRM and CMT3 methyltransferases in RNA-directed DNA methylation. Curr. Biol. 13, 2212-2217. doi: 10.1016/j.cub.2003.11.052

Cao, X., and Jacobsen, S. E. (2002). Locus-specific control of asymmetric and CpNpG methylation by the DRM and CMT3 methyltransferase genes. Proc. Natl. Acad. Sci. U.S.A 99, 16491-16498. doi: 10.1073/pnas.162371599

Chan, S. W., Henderson, I. R., and Jacobsen, S. E. (2005). Gardening the genome: DNA methylation in Arabidopsis thaliana. Nat. Rev. Genet. 6, 351-360. doi: $10.1038 / \mathrm{nrg} 1601$

Cokus, S. J., Feng, S., Zhang, X., Chen, Z., Merriman, B., Haudenschild, C. D., et al. (2008). Shotgun bisulphite sequencing of the Arabidopsis genome reveals DNA methylation patterning. Nature 452, 215-219. doi: 10.1038/nature06745

Gao, Z., Liu, H. L., Daxinger, L., Pontes, O., He, X., Qian, W., et al. (2010). An RNA polymerase II- and AGO4-associated protein acts in RNA-directed DNA methylation. Nature 465, 106-109. doi: 10.1038/nature09025

Gruntman, E., Qi, Y., Slotkin, R. K., Roeder, T., Martienssen, R. A., and Sachidanandam, R. (2008). Kismeth: analyzer of plant methylation states through bisulfite sequencing. BMC Bioinformatics 9:371. doi: 10.1186/1471-2105-9-371

Hajdukiewicz, P., Svab, Z., and Maliga, P. (1994). The small, versatile pPZP family of Agrobacterium binary vectors for plant transformation. Plant Mol. Biol. 25, 989-994. doi: 10.1007/BF00014672

Hartung, F., and Schiemann, J. (2014). Precise plant breeding using new genome editing techniques: opportunities, safety and regulation in the EU. Plant J. 78, 742-752. doi: 10.1111/tpj.12413

He, X. J., Hsu, Y. F., Zhu, S., Liu, H. L., Pontes, O., Zhu, J., et al. (2009). A conserved transcriptional regulator is required for RNA-directed DNA methylation and plant development. Genes Dev. 23, 2717-2722. doi: 10.1101/gad. 1851809

Henderson, I. R., and Jacobsen, S. E. (2007). Epigenetic inheritance in plants. Nature 447, 418-424. doi: 10.1038/nature05917

Igarashi, A., Yamagata, K., Sugai, T., Takahashi, Y., Sugawara, E., Tamura, A., et al. (2009). Apple latent spherical virus vectors for reliable and effective virus-induced gene silencing among a broad range of plants including tobacco, tomato, Arabidopsis thaliana, cucurbits, and legumes. Virology 386, 407-416. doi: 10.1016/j.virol.2009.01.039

Jones, L., Hamilton, A. J., Voinnet, O., Thomas, C. L., Maule, A. J., and Baulcombe, D. C. (1999). RNA-DNA interactions and DNA methylation in post-transcriptional gene silencing. Plant Cell 11, 2291-2301. doi: 10.1105/tpc.11.12.2291

Jones, L., Ratcliff, F., and Baulcombe, D. C. (2001). RNA-directed transcriptional gene silencing in plants can be inherited independently of the RNA trigger and requires Met1 for maintenance. Curr. Biol. 11, 747-757. doi: 10.1016/S09609822(01)00226-3

Kanazawa, A., Inaba, J., Shimura, H., Otagaki, S., Tsukahara, S., Matsuzawa, A., et al. (2011). Virus-mediated efficient induction of epigenetic modifications of endogenous genes with phenotypic changes in plants. Plant J. 65, 156-168. doi: 10.1111/j.1365-313X.2010.04401.x

Kanazawa, A., O’Dell, M., and Hellens, R. P. (2007). The binding of nuclear factors to the as-1 element in the CaMV 35S promoter is affected by cytosine methylation in vitro. Plant Biol. 9, 435-441. doi: 10.1055/s-2006-924633

Kon, T., and Ikegami, M. (2009). RNA silencing in plants and its suppression by plant viruses. CAB Rev. 4, 1-16. doi: 10.1079/PAVSNNR20094064

Kon, T., Rojas, M. R., Abdourhamane, I. K., and Gilbertson, R. L. (2009). The role and interactions of begomoviruses and satellite DNAs associated with okra leaf curl disease in Mali, West Africa. J. Gen. Virol. 90, 1001-1013. doi: 10.1099/vir.0.008102-0

Law, J. A., Ausin, I., Johnson, L. M., Vashisht, A. A., Zhu, J. K., Wohlschlegel, J. A., et al. (2010). A protein complex required for polymerase $\mathrm{V}$ transcripts and RNA-directed DNA methylation in Arabidopsis. Curr. Biol. 20, 951-956. doi: 10.1016/j.cub.2010.03.062

Law, J. A., and Jacobsen, S. E. (2010). Establishing, maintaining and modifying DNA methylation patterns in plants and animals. Nat. Rev. Genet. 11, 204-220. doi: $10.1038 / \operatorname{nrg} 2719$ 
Le Gall, O., Sanfaçon, H., Ikegami, M., Iwanami, T., Jones, T., Karasev, A., et al. (2007). Cheravirus and Sadwavirus: two unassigned genera of plant positivesense single-stranded RNA viruses formerly considered atypical members of the genus Nepovirus (family Comoviridae). Arch. Virol. 152, 1767-1774. doi: 10.1007/s00705-007-1015-0

Li, C., Sasaki, N., Isogai, M., and Yoshikawa, N. (2004). Stable expression of foreign proteins in herbaceous and apple plants using apple latent spherical virus RNA2 vectors. Arch. Virol. 149, 1541-1558. doi: 10.1007/s00705-004-0310-2

Li, C., Yoshikawa, N., Takahashi, T., Ito, T., Yoshida, K., and Koganezawa, H. (2000). Nucleotide sequence and genome organization of apple latent spherical virus: a new virus classified into the family Comoviridae. J. Gen. Virol. 81, 541-547.

Liu, S., Yeh, C. T., Ji, T., Ying, K., Wu, H., Tang, H. M., et al. (2009). Mu transposon insertion sites and meiotic recombination events co-localize with epigenetic marks for open chromatin across the maize genome. PLoS Genet. 5:e1000733. doi: 10.1371/journal.pgen.1000733

Liu, Y. L., Schiff, M., and Dinesh-Kumar, S. P. (2002). Virus-induced gene silencing in tomato. Plant J. 31, 777-786. doi: 10.1046/j.1365-313X.2002.01394.x

Molnar, A., Melnyk, C. W., Bassett, A., Hardcastle, T. J., Dunn, R., and Baulcombe, D. C. (2010). Small silencing RNAs in plants are mobile and direct epigenetic modification in recipient cells. Science 328, 872-875. doi: 10.1126/science.1187959

Morita, Y., Saito, R., Ban, Y., Tanikawa, N., Kuchitsu, K., Ando, T., et al. (2012). Tandemly arranged chalcone synthase a genes contribute to the spatially regulated expression of siRNA and the natural bicolor floral phenotype in Petunia hybrida. Plant J. 70, 739-749. doi: 10.1111/j.1365-313X.2012.04908.x

Pontes, O., Li, C. F., Costa Nunes, P., Haag, J., Ream, T., Vitins, A., et al. (2006). The Arabidopsis chromatin-modifying nuclear siRNA pathway involves a nucleolar RNA processing center. Cell 126, 79-92. doi: 10.1016/j.cell.2006.05.031

Sasaki, S., Yomagishi, N., and Yoshikawa, N. (2011). Efficient virus-induced gene silencing in apple, pear and Japanese pear using apple latent spherical virus vectors. Plant Methods 7:15. doi: 10.1186/1746-4811-7-15

Senthil-Kumar, M., and Mysore, K. S. (2011). New dimensions for VIGS in plant functional genomics. Trends Plant Sci. 16, 656-665. doi: 10.1016/j.tplants.2011.08.006

Vaghchhipawala, Z., Rojas, C. M., Senthil-Kumar, M., and Mysore, K. S. (2011). Agroinoculation and agroinfiltration: simple tools for complex gene function analyses. Methods Mol. Biol. 678, 65-76. doi: 10.1007/978-1-60761-682-5_6

van der Meer, I. M., Spelt, C. E., Mol, J. N., and Stuitje, A. R. (1990). Promoter analysis of the chalcone synthase (chsA) gene of Petunia hybrida: a 67 bp promoter region directs flower-specific expression. Plant Mol. Biol. 15, 95-109. doi: $10.1007 / \mathrm{BF} 00017727$

Vaucheret, H. (2006). Post-transcriptional small RNA pathways in plants: mechanisms and regulations. Genes Dev. 20, 759-771. doi: 10.1101/gad.1410506
Wierzbicki, A. T., Haag, J. R., and Pikaard, C. S. (2008). Noncoding transcription by RNA polymerase Pol IVb/Pol V mediates transcriptional silencing of overlapping and adjacent genes. Cell 135, 635-648. doi: 10.1016/j.cell.2008. 09.035

Wierzbicki, A. T., Ream, T. S., Haag, J. R., and Pikaard, C. S. (2009). RNA polymerase $\mathrm{V}$ transcription guides ARGONAUTE4 to chromatin. Nat. Genet. 41, 630-634. doi: 10.1038/ng.365

Yaegashi, H., Yamatsuta, T., Takahashi, T., Li, C., Isogai, M., Kobori, T., et al. (2007) Characterization of virus-induced gene silencing in tobacco plants infected with apple latent spherical virus. Arch. Virol. 152, 1839-1849. doi: 10.1007/s00705007-1011-4

Yamagishi, N., Sasaki, S., Yamagata, K., Komori, S., Nagase, M., Wada, M., et al. (2011). Promotion of flowering and reduction of the generation time in apple seedlings by ectopical expression of the Arabidopsis thaliana FT gene using the apple latent spherical virus vector. Plant Mol. Biol. 75, 193-204. doi: 10.1007/s11103-010-9718-0

Yamagishi, N., and Yoshikawa, N. (2009). Virus-induced gene silencing in soybean seeds and the emergence stage of soybean plants with apple latent spherical virus vectors. Plant Mol. Biol. 71, 15-24. doi: 10.1007/s11103-0099505-y

Zaratiegui, M., Irvine, D., and Martienssen, R. (2007). Noncoding RNAs and gene silencing. Cell 128, 763-776. doi: 10.1016/j.cell.2007. 02.016

Zemach, A., McDaniel, I. E., Silva, P., and Zilberman, D. (2010). Genome-wide evolutionary analysis of eukaryotic DNA methylation. Science 328, 916-919. doi: $10.1126 /$ science. 1186366

Conflict of Interest Statement: The authors declare that the research was conducted in the absence of any commercial or financial relationships that could be construed as a potential conflict of interest.

Received: 02 September 2014; accepted: 21 October 2014; published online: 10 November 2014.

Citation: Kon T and Yoshikawa N (2014) Induction and maintenance of DNA methylation in plant promoter sequences by apple latent spherical virus-induced transcriptional gene silencing. Front. Microbiol. 5:595. doi: 10.3389/fmicb.2014. 00595

This article was submitted to Virology, a section of the journal Frontiers in Microbiology. Copyright (c) 2014 Kon and Yoshikawa. This is an open-access article distributed under the terms of the Creative Commons Attribution License (CC BY). The use, distribution or reproduction in other forums is permitted, provided the original author(s) or licensor are credited and that the original publication in this journal is cited, in accordance with accepted academic practice. No use, distribution or reproduction is permitted which does not comply with these terms. 